\title{
LES of the Cambridge Stratified Swirl Burner using a Sub-grid pdf Approach
}

\author{
T. Brauner ${ }^{1}$ - W. P. Jones ${ }^{1}$ - A. J. Marquis ${ }^{1}$
}

Received: 29 October 2015 / Accepted: 10 February 2016 / Published online: 8 March 2016

(C) The Author(s) 2016. This article is published with open access at Springerlink.com

\begin{abstract}
The sub-grid scale probability density function equation is rearranged in order to separate the resolved and sub-grid-scale ( $\mathrm{sgs}$ ) contributions to the $s g s$ mixing term. This allows modelling that is consistent with the limiting case of negligible sub-grid scale variations, a property required for applications to laboratory premixed flames. The new method is applied to the Cambridge Stratified Swirl Burner for 6 operating conditions, 2 isothermal and 4 burning, with varying degrees of swirl and mixture stratification. The simulations are performed with the Large Eddy Simulation (LES) code BOFFIN in which the modelled $p d f$ transport equation is solved using the Eulerian stochastic field method. Eight stochastic fields are used to account for the influence of the sub-grid fluctuations and the chemistry is modelled with a reduced version of the GRI 3.0 mechanism for methane involving 19 species and 15 reaction steps. The simulated velocities for both the isothermal and burning cases show good agreement with the experimental data. The measured temperature and major species profiles are also reproduced to a good accuracy.
\end{abstract}

Keywords Stratified Flames · Large eddy simulation · Sub-grid scale pdf equation · Stochastic Fields

W. P. Jones

w.jones@imperial.ac.uk

1 Mechanical Engineering Department, Imperial College London, London SW7 2AZ, UK 


\section{Introduction}

Legislation governing pollutant emissions has promoted a development trend away from high temperature stoichiometric combustion towards lean premixed combustion. This mode delivers lower temperatures and complete combustion, resulting in lower emissions of, for example carbon monoxide and oxides of nitrogen. Time and space requirements on such combustion devices may pose constraints that influence the premixing of the fuel and oxidiser, resulting in an inhomogeneous mixture prior to combustion; this may lead to combustion taking place under partially premixed conditions. In the absence of non-premixed combustion this may also be referred to as stratified combustion, where the equivalence ratio of the mixture exhibits a spatial gradient.

In order to further develop such systems it is important to investigate the fundamental properties of stratified combustion in simple test cases such as the stratified V-Flame [1-3] and gain the ability to accurately predict the behaviour of flames under these conditions, as their properties remain relatively poorly characterised and understood compared to other combustion regimes [4]. Findings from such studies have shown deviations from homogeneous systems with increased lean flammability limits, increased variations in burning velocity, higher flame propagation rates along with increased flame wrinkling and flame thinning. However, typical gas turbine combustion chambers for example feature complex geometries with a range of turbulent flows and physical conditions and generally use swirl as a means of improving mixing, as well as stabilising and shortening the flames.

The Cambridge Stratified Swirl Burner [5, 6] provides a flame series that allows for the numerical investigation of flames operating at laboratory conditions closer to those of an industrial configuration, while providing detailed experimental data against which to validate models. Large eddy simulation is now a practical tool for modelling the interaction of turbulence and chemistry and previous numerical investigations into the non-swirling flames of the Cambridge Stratified Swirl Burner have been conducted, using various flamelet approaches, by amongst others [7] and [8]. The flames considered, while turbulent, have relatively low Reynolds numbers with the consequence that $s g s$ fluctuations are often negligible over substantial regions of the flames. In these circumstances it is necessary that any model behaves correctly in the limit of $s g s$ variations approaching zero, the so-called DNS limit. The present work utilises the sub-grid scale probability density function ( $p d f$ ) approach, which is rearranged so that the resolved and sgs contributions are separated. This allows the unclosed terms to be modelled so that they approach zero as the sgs fluctuations approach zero such that the $p d f$ approaches a Dirac deltafunction, i.e. the DNS limit. The resulting closed form of the $p d f$ equation is then solved using the Eulerian stochastic fields, which has been implemented in the block-structured finite-volume code BOFFIN-LES. The $p d f$ and stochastic field method do not involve any assumptions related to any particular combustion regime and the method has previously successfully been applied to a range of non-premixed, partially premixed and fully premixed flames including, for example, auto-ignition [9], the Sandia Flame Series [10], spark ignition [11], partially premixed combustion [12] and recently to an industrial gas-turbine [13].

The aim of the present work is to numerically investigate the influence of mixture stratification on turbulent swirling flames and further validate the $p d f$ equation / stochastic field method in the context of premixed stratified combustion. 


\section{Large Eddy Simulation and the Stochastic Field Method}

The filtered continuity and Navier-Stokes equations and the filtered form of the transport equation for any scalar, $\phi_{\alpha}$ such as enthalpy or species' mass fraction are derived by applying a density weighted 'top-hat' filter resulting in the following:

$$
\begin{gathered}
\frac{\partial \bar{\rho}}{\partial t}+\frac{\partial \bar{\rho} \tilde{u_{i}}}{\partial x_{i}}=0 \\
\frac{\partial \bar{\rho} u_{i}}{\partial t}+\frac{\partial \bar{\rho} \tilde{u_{i}} \tilde{u_{j}}}{\partial x_{j}}=-\frac{\partial \bar{p}}{\partial x_{i}}+\frac{\partial}{\partial x_{j}} \bar{\mu}\left(\frac{\partial \tilde{u_{i}}}{\partial x_{j}}+\frac{\partial \tilde{u_{j}}}{\partial x_{i}}-\frac{2}{3} \frac{\partial \tilde{u_{k}}}{\partial x_{k}} \delta_{i j}\right)+\frac{\partial \tau_{i j}}{\partial x_{j}} \\
\frac{\partial \bar{\rho} \tilde{\phi_{\alpha}}}{\partial t}+\frac{\partial \bar{\rho} \tilde{u_{j}} \tilde{\phi_{\alpha}}}{\partial x_{j}}=\frac{\partial}{\partial x_{j}}\left[\bar{\rho} D \frac{\partial \tilde{\phi_{\alpha}}}{\partial x_{j}}\right]+\overline{\rho \dot{\omega}_{\alpha}\left(\phi_{\beta}, \mathrm{T}\right)}-\frac{\partial J_{\alpha, j}^{s g s}}{\partial x_{j}}
\end{gathered}
$$

The sub-grid scale (sgs) stress, defined as $\tau_{i j}=-\bar{\rho}\left(\widetilde{u_{i} u_{j}}-\tilde{u_{i}} \tilde{u_{j}}\right)$ is closed using a dynamic version of the Smagorinsky model [14]:

$$
\mu_{s g s}=\bar{\rho}\left(C_{S} \Delta\right)^{2}\left\|\tilde{e}_{i j}\right\|
$$

where $\left\|\tilde{e}_{i j}\right\| \equiv \sqrt{2 \tilde{e}_{i j} \tilde{e}_{i j}}$ is the Frobenius norm of the resolved rate of strain tensor, $\tilde{e}_{i j}=0.5\left(\frac{\partial \tilde{u}_{i}}{\partial x_{j}}+\frac{\partial \tilde{u}_{j}}{\partial x_{i}}\right)$. The filter width is taken as the cube root of the local grid cell volume and the parameter $C_{s}$ is obtained through the dynamic procedure of Piomelli and Liu [15]. As with the viscous stress the isotropic part of the $\operatorname{sgs}$ stress is adsorbed into the pressure.

The main difficulty in LES of combustion processes is the closure of the filtered chemical source term appearing in Equation (3). For this it is possible to use a one-point joint filtered $p d f$ for all the scalar quantities required to describe the reaction. The transport equation of such a $p d f$ provides a means of describing the temporal and spatial variation of the scalars one-point statistics and has the advantage that the chemical source term appears in closed form. Using the filtering operation and following Gao and O'Brien [16] unweighted and density weighted $s g s$ (or filtered) $p d f s$ for the $N_{s}$ scalar quantities needed to describe reaction (the number of species considered plus enthalpy) can be defined:

$$
\begin{aligned}
& \bar{P}(\underline{\psi} ; \mathbf{x}, t)=\int_{\Omega} \mathscr{F}\left(\underline{\psi} ; \mathbf{x}^{\prime}, t\right) G\left(\mathbf{x}-\mathbf{x}^{\prime}, \Delta\right) d \mathbf{x}^{\prime} \\
& \tilde{P}(\underline{\psi} ; \mathbf{x}, t)=\int_{\Omega} \frac{\rho(\underline{\psi})}{\bar{\rho}} \mathscr{F}\left(\underline{\psi} ; \mathbf{x}^{\prime}, t\right) G\left(\mathbf{x}-\mathbf{x}^{\prime}, \Delta\right) d \mathbf{x}^{\prime}
\end{aligned}
$$

where $\mathscr{F}(\underline{\psi} ; \mathbf{x}, t)=\prod_{\alpha=1}^{N_{s}} \delta\left(\psi_{\alpha}-\phi_{\alpha}(\mathbf{x}, t)\right)$ is a fine-grained $p d f$ and $\underline{\psi}$ represents the phase or sample space of the scalar quantities $\phi$. Providing the filter in Equation (4) is positive definite then the $p d f, P$ describes the probability of $\psi<\phi \leq \psi+d \underline{\psi}$ arising within the filter 'volume'. An exact evolution equation for this quantity can then be derived, e.g. [16-18], from the appropriate conservation equations by standard methods.

The flames to be considered in the present study have relatively low Reynolds numbers and $s g s$ fluctuations can be very small or zero in some regions of the flow. In these circumstances it is important that the sgs-pdf behaves correctly in the limit of small and zero sgs 
fluctuations. For this reason the derivation and modelling of the evolution equation for $P$ is considered further in the present paper and the exact equation for $\tilde{P}$ is thus written in the form:

$$
\begin{aligned}
& \frac{\partial \bar{\rho} \tilde{P}(\underline{\psi})}{\partial t}+\frac{\partial \bar{\rho} \tilde{u}_{j} \tilde{P}(\underline{\psi})}{\partial x_{j}}-\frac{\partial}{\partial x_{j}}\left(\frac{\mu}{\sigma} \frac{\partial \bar{P}(\underline{\psi})}{\partial x_{j}}\right)+\sum_{\alpha=1}^{N} \sum_{\beta=1}^{N} \frac{\mu}{\sigma} \frac{\partial \bar{\phi}_{\alpha}}{\partial x_{i}} \frac{\partial \bar{\phi}_{\beta}}{\partial x_{i}} \frac{\partial^{2} \bar{P}(\underline{\psi})}{\partial \psi_{\alpha} \partial \psi_{\beta}} \\
& +\sum_{\alpha=1}^{N} \frac{\partial}{\partial \psi_{\alpha}} \bar{\rho} \dot{\omega}_{\alpha}(\underline{\psi}) \tilde{P}(\underline{\psi})= \\
& -\frac{\partial\left(\overline{\rho u_{j} \mathscr{F}(\underline{\psi})}-\bar{\rho} \tilde{u}_{j} \tilde{P}(\underline{\psi})\right)}{\partial x_{j}} \\
& -\sum_{\alpha=1}^{N} \sum_{\beta=1}^{N} \frac{\mu}{\sigma}\left(\frac{\partial^{2}}{\partial \psi_{\alpha} \partial \psi_{\beta}}\left(\overline{\frac{\partial \phi_{\alpha}}{\partial x_{i}} \frac{\partial \phi_{\beta}}{\partial x_{i}} \mathscr{F}(\underline{\psi})}\right)-\frac{\partial \bar{\phi}_{\alpha}}{\partial x_{i}} \frac{\partial \bar{\phi}_{\beta}}{\partial x_{i}} \frac{\partial^{2} \bar{P}(\underline{\psi})}{\partial \psi_{\alpha} \partial \psi_{\beta}}\right)
\end{aligned}
$$

where $\sigma$ is the Prandtl or Schmidt number, assumed constant and equal and where the influence of $s g s$ fluctuations on the viscosity, $\mu$ have been neglected. It is worth noting that $\tilde{P}$ like the Dirac delta function, $\delta(\ldots)$ is a generalised function, defined only in terms of its integral properties; the terms in Equation (5) are defined only when written in integrated form. In Equation (5) the resolved contributions to the advection and the $s g s$ molecular diffusion/mixing terms have been added to both sides of the equation with the consequence that the rhs of the equation involves contributions from sgs fluctuations only; identically zero if sgs variations are zero, i.e. the variations in all relevant quantities over the filter volume are negligible. The final term on the rhs of the equation, the diffusive mixing term, approaches zero as the variation in the $\phi$ 's over the filter 'volume' become linear. The shape of the $p d f$ in this latter limit and with velocity and density constant over the filter 'volume' depends on the choice of filter. For the 'top-hat' filter presently used the $p d f$ becomes uniformly distributed between the minimum and maximum values arising at the 'edges' of the filter 'volume'. In the DNS limit where the governing equations are solved without approximation the filtering operation has negligible influence and the $p d f$ reduces to the fine-grained $p d f$.

The lhs of the equation involves only known terms whereas the rhs requires modelling in LES. This separation of terms is common practice for the advection term but to date it does not seem to have been applied to the diffusive mixing term. A further consequence of the approach is that the $\tilde{P}$ equation becomes exact in the limit that $s g s$ variations are zero. For example the equation for the second moment of a scalar becomes:

$$
\rho \frac{\partial \phi^{2}}{\partial t}+\rho u_{i} \frac{\partial \phi^{2}}{\partial x_{i}}=\frac{\partial}{\partial x_{i}}\left(\frac{\mu}{\sigma} \frac{\partial \phi^{2}}{\partial x_{i}}\right)-2 \frac{\mu}{\sigma}\left(\frac{\partial \phi}{\partial x_{i}}\right)^{2}+2 \rho \phi \dot{\omega}_{\alpha}(\underline{\phi})
$$

The right hand side of equation (5) is approximated by a simple gradient closure directly analogous to the Smagorinsky model:

$$
\overline{\rho(\underline{\psi}) \mathscr{F}(\underline{\psi}) u_{j}}-\bar{\rho} \tilde{u}_{j} \tilde{P}=-\frac{\mu_{s g s}}{\sigma_{s g s}} \frac{\partial \tilde{P}}{\partial x_{j}}
$$

where $\sigma_{s g s}$ is a constant turbulent Prandtl/Schmidt number assigned the value 0.7, [19]. The final term in equation (5) represents sub-grid scale mixing and describes the effect 
of molecular diffusion on $\tilde{P}(\psi)$. In the present work the Linear Mean Square estimation (LMSE) closure [20-22] is adopted. Thus the final term in equation (5) is replaced by:

$$
\frac{C_{d}}{\tau_{s g s}} \sum_{\alpha=1}^{N_{s}} \frac{\partial}{\partial \psi_{\alpha}}\left[\left(\psi_{\alpha}-\tilde{\phi}_{\alpha}(\mathbf{x}, t)\right) \bar{\rho} \tilde{P}(\underline{\psi})\right]
$$

It is to be noted that the LMSE model does not satisfy the limiting property of approaching zero as the variation in the $\phi$ 's over the filter volume become linear; the construction of such a model appears difficult and is beyond the scope of the present paper. Also the model for the sgs diffusive mixing term should be such that the $p d f$ collapses instantaneously to a Dirac $\delta$-function in the limit of the sgs variations approaching zero, i.e. in well resolved regions of the flow or where the DNS limit is achieved. In this limiting case the time scale $\tau_{s g s}$ should tend to zero. The $s g s$ viscosity provides a measure of $s g s$ fluctuations and this can be used to ensure the desired limiting behaviour. A dynamic model is used to determine $C_{s}$ and a zero value of this implies no $s g s$ variations. Thus analogous to [12] a modified time scale is employed:

$$
\tau_{s g s}=\frac{\bar{\rho} \Delta^{2}}{\mu_{s g s}}\left(1-\exp \left(-\mathscr{R}^{2}\right)\right)
$$

where $\mathcal{R}$ is a sgs turbulence Reynolds number defined as $\mu_{s g s} / \mu$. This modified time scale has the desired limiting behaviour $\tau_{s g s} \rightarrow 0$ as $\mu_{s g s} \rightarrow 0$ with the consequence that the $p d f$ will tend towards a Dirac $\delta$-function. The model provides increased micro-mixing in regions where the $s g s$ fluctuations are small although the modification becomes effective only if the $s g s$ viscosity is comparable with or small compared to the molecular viscosity.

The closed form of equation (5) describing the evolution of the $p d f$ with closures (6)(8) is solved using the Eulerian stochastic field method, [9]. $\tilde{P}(\psi)$ is represented by an ensemble of $N_{s}$ stochastic fields for each of the $N$ scalars namely $\xi_{\alpha}^{\bar{n}}(\mathbf{x}, t)$ with $1 \leq n \leq N_{s}$; $1 \leq \alpha \leq N$. In the present work the Itô formulation of the stochastic integral is adopted and the stochastic fields thus evolve according to:

$$
\begin{aligned}
\bar{\rho} \mathrm{d} \xi_{\alpha}^{n}= & -\bar{\rho} \tilde{u}_{i} \frac{\partial \xi_{\alpha}^{n}}{\partial x_{i}} \mathrm{~d} t+\frac{\partial}{\partial x_{i}}\left[\left(\frac{\mu}{\sigma}+\frac{\mu_{s g s}}{\sigma_{s g s}}\right) \frac{\partial \xi_{\alpha}^{n}}{\partial x_{i}}\right] \mathrm{d} t+\sqrt{2 \bar{\rho} \frac{\mu_{s g s}}{\sigma_{s g s}}} \frac{\partial \xi_{\alpha}^{n}}{\partial x_{j}} \mathrm{~d} W_{i}^{n} \\
& -\frac{C_{d} \bar{\rho}}{2 \tau_{s g s}}\left(\xi_{\alpha}^{n}-\tilde{\phi}_{\alpha}\right) \mathrm{d} t+\bar{\rho} \dot{\omega}_{\alpha}^{n}\left(\underline{\xi}^{n}\right) \mathrm{d} t
\end{aligned}
$$

where $d W_{i}^{n}$ represents increments of a vector Wiener process, different for each field but independent of the spatial location $\mathbf{x}$. This stochastic term has no effect on the first moments (or filtered values) of $\xi_{\alpha}^{n}$ but exerts a strong influence on the shape and 'width' of the $p d f$. It is worth noting, that unlike earlier formulations, the stochastic term now depends only on the sub-grid viscosity and is thus zero in the event of no sub-grid variations. The Wiener process is approximated by time-step increments $\eta_{i}^{n} \sqrt{\mathrm{d} t}$, where $\eta_{i}^{n}$ is a $\{-1,+1\}$ dichotomic random vector. The solutions of equation (9) preserve any bound properties of the scalar in question as the gradient of the scalar will tend to zero as the value of the scalar approaches extrema values, [23] and therefore the stochastic contribution will tend to zero. The solutions for each field will satisfy all the mass conservation and bound properties of the modelled $p d f$ equation (5). For each field, for example, the species mass fractions will remain positive and will sum to unity. For a large number of fields the filtered value of the stochastic term will tend to zero. The solutions of the stochastic field equation (9) form an equivalent stochastic system (both sets have the same one-point $p d f$, [24]) smooth on the scale of the filter width. All the moments resulting from the stochastic differential equations 
and from the direct solutions of the modelled form of equation (5) will be identical. All of the moments of the scalar fields were obtained by averaging over the stochastic fields as appropriate. For example all first moments were obtained from:

$$
\tilde{\phi}_{\alpha}=\frac{1}{N} \sum_{n=1}^{N} \xi_{\alpha}^{n}
$$

A form similar to Equation (9) is proposed in [25]. A Reynolds decomposition is applied to the exact equation for $\tilde{P}$, thus effectively separating out the turbulent contribution to diffusive mixing. While the proposal of [25] is consistent with an entirely laminar flow when extended to LES, it does not ensure that the $p d f$ collapses to a Dirac delta function in any region of a turbulent flame where the flow becomes laminar, i.e. where sgs fluctuations become negligible.

\section{Cambridge Stratified Swirl Burner and Computational Setup}

The burner configuration under investigation is the Cambridge Stratified Swirl Burner. The results of the experiments are reported in $[5,6,26]$. The burner consists of a central bluff body surrounded by two concentric annular channels and a co-flow assembly to prevent entrainment of ambient air. A schematic of the burner is shown in Fig. 1, including the approximate location of the flame brush indicated by the dashed lines. Each annulus supplies a stream of premixed methane/air mixture, the equivalence ratios of which can be varied. The average equivalence ratio is maintained at $\phi_{\text {avg }}=0.75$. The outer annulus can additionally introduce swirl with different degrees of swirl to flow ratio (SFR). The SFR is defined as the ratio of flow rate passing through the swirler passage to the axial flow rate. The flow in the outer annulus has a mean velocity of $U_{o}=18.7 \mathrm{~m} / \mathrm{s}$, in the inner annulus of $U_{i}=8.7 \mathrm{~m} / \mathrm{s}$ and the co-flow air of $U_{c o}=0.4 \mathrm{~m} / \mathrm{s}$. The Reynolds numbers based on these velocities and the annuli exits are $R e_{o}=11500$ and $R e_{i}=5960$ for the outer and inner annuli respectively. The burner is unconfined and the flame is stabilised at the central

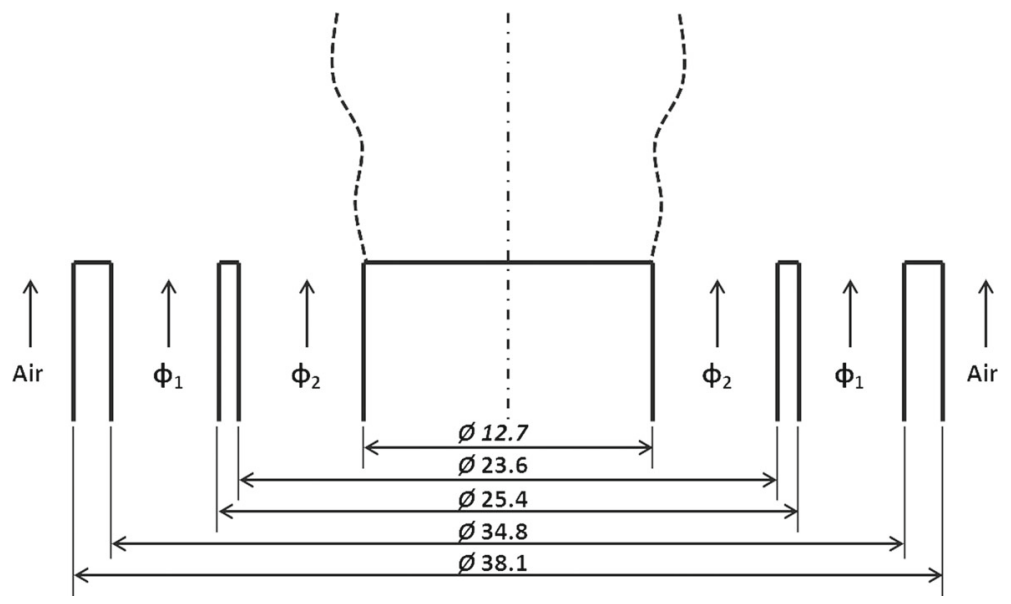

Fig. 1 Schematic of the Cambridge Stratified Swirl Burner geometry 
Table 1 Stratification-ratios and swirl-flow-ratios for different operating conditions

\begin{tabular}{lll}
\hline Case & $\phi_{\text {inner }} / \phi_{\text {outer }}$ & SFR \\
\hline cSwB1 & isothermal & 0.0 \\
cSwB3 & isothermal & 0.33 \\
SwB1 & $0.75 / 0.75=1.0$ & 0.0 \\
SwB3 & $0.75 / 0.75=1.0$ & 0.33 \\
SwB5 & $1.0 / 0.5=2.0$ & 0.0 \\
SwB7 & $1.0 / 0.5=2.0$ & 0.33 \\
\hline
\end{tabular}

bluff body. The Damköhler number is estimated, [27] to be between 0.89 and 1.24 and the Karlowitz number between 93 and 215. Under these conditions the flame falls into the thin reaction zone regime. The configurations investigated in the work presented here are the non-swirling and highly swirling, premixed and moderately stratified cases, the operating conditions of which are summarised in Table 1.

Extensive velocity, temperature and mass fraction measurements are available at increments of $10 \mathrm{~mm}$ above the burner exit. Velocity measurements were obtained with Laser Doppler Anemometry (LDA) as well as Particle Image Velocimetry (PIV), while Rayleigh and Raman scattering line measurements provide the temperature and major species respectively. Flows involving an annular bluff body are sensitive to the alignment of the ducts and even minor deviations can result in asymmetric flow behaviour. This is reflected in the measured profiles and was also reported in [7].

The computational domain used includes $25 \mathrm{~mm}$ of the annuli upstream of the bluff body in order to better capture the flow conditions at the burner exit. The domain extends $300 \mathrm{~mm}$ downstream of the bluff body in the axial direction and $200 \mathrm{~mm}$ in the radial directions. The mesh used, slices of which are shown in Fig. 2, consists of approximately four million nodes divided into 163 blocks, resulting in an average mesh spacing of about $0.3 \mathrm{~mm}$ in the vicinity of the bluff body. The boundaries consist of two inlets (three if the large, low velocity coflow is included), zero-gradient outflow conditions, adiabatic and Neumann no slip conditions with a wall function at the bluff body and annuli walls and free slip conditions on all other boundaries.

To characterise the inflow conditions a digital inflow generator is used to model the effect of the turbulence at the inner and outer annuli exits of the burner. Experimental velocity
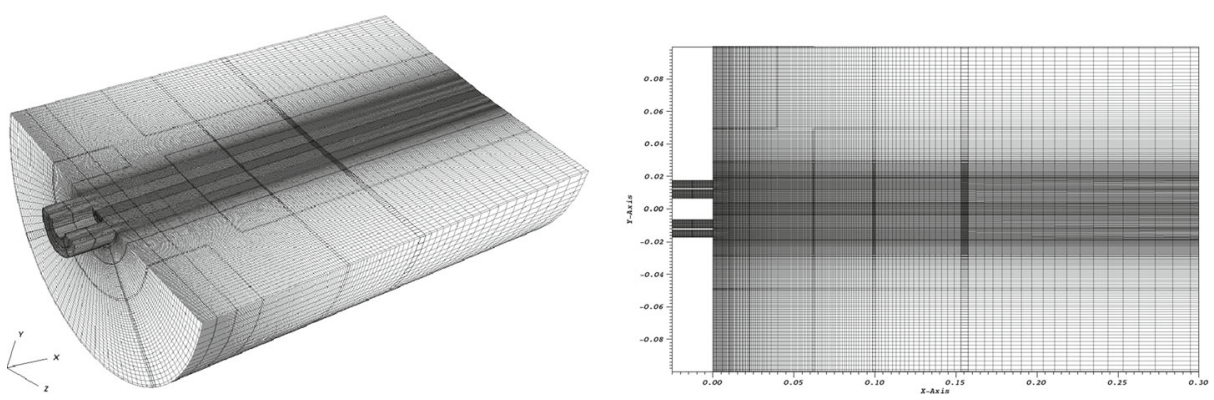

Fig. 2 Slices through the computational mesh 

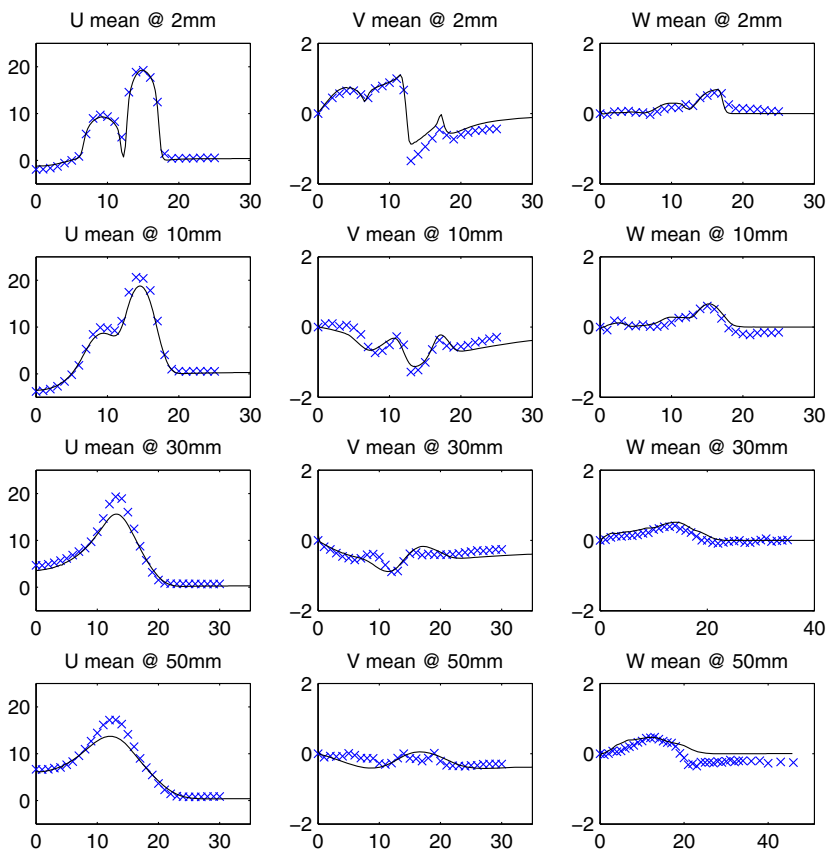

(a) Profiles of mean velocity components
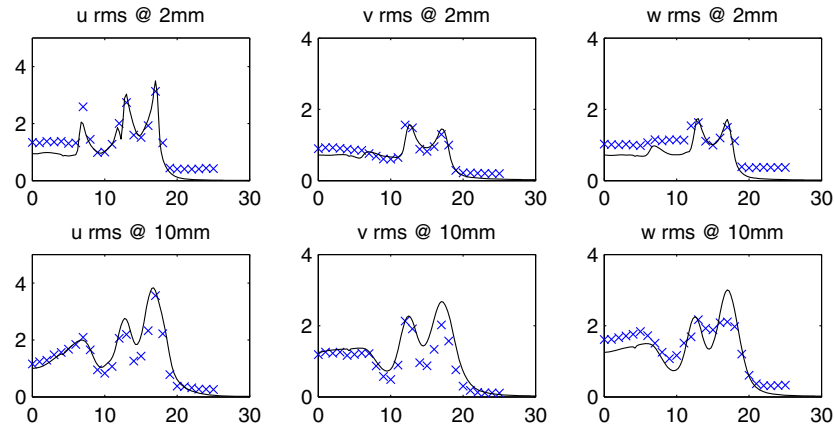

w rms @10mm
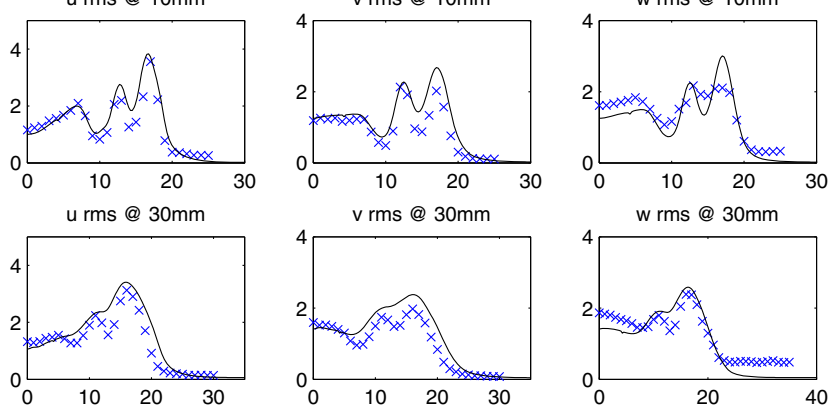

u rms@ @ $50 \mathrm{~mm}$
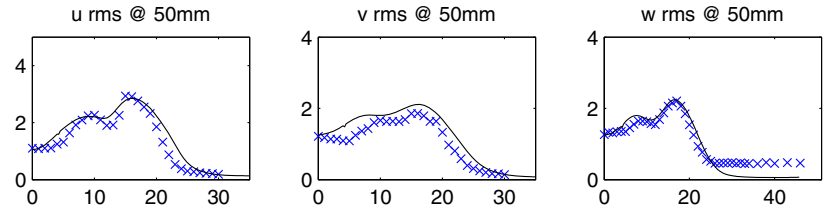

(b) Profiles of rms velocity components

Fig. 3 Comparison of axial (U), radial (V) and tangential (W) mean and rms (u,v,w) velocities for the isothermal, non-swirling case, cSwB1 

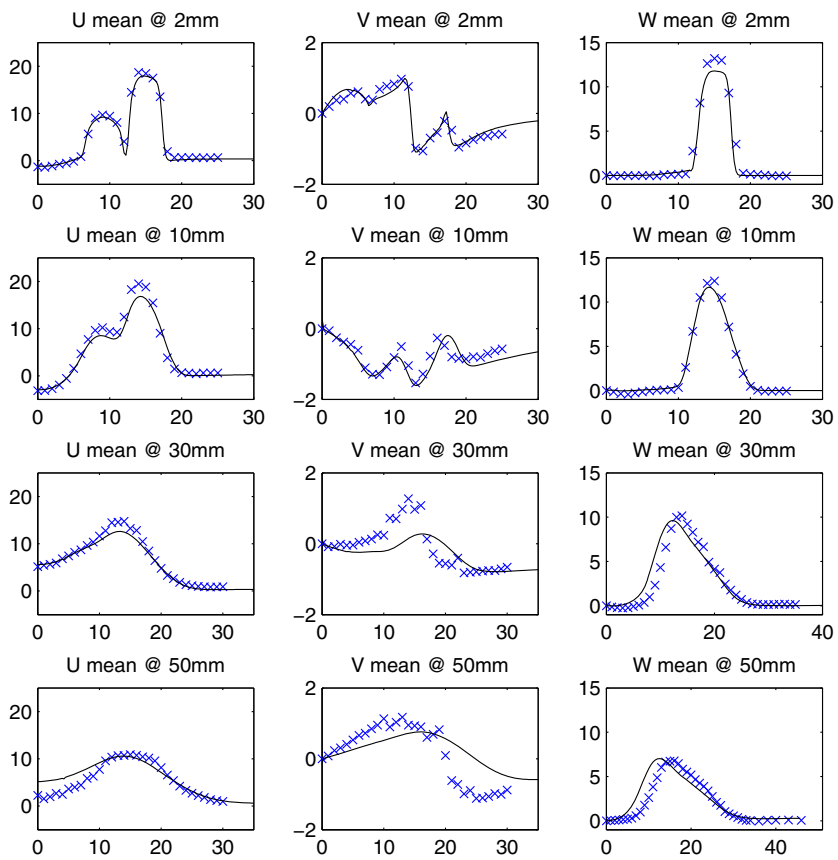

(a) Profiles of mean velocity components
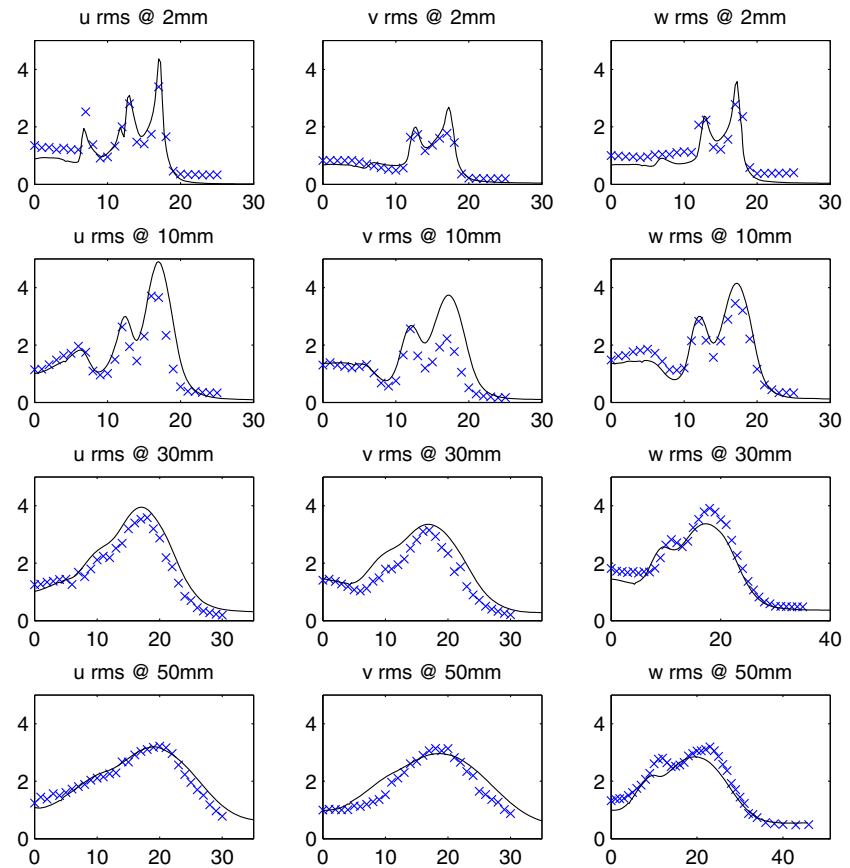

(b) Profiles of rms velocity components

Fig. 4 Comparison of axial (U), radial (V) and tangential (W) mean and rms (u,v,w) velocities for the isothermal, highly swirling case with $S F R=0.33, \mathrm{cSwB} 3$ 
Fig. 5 Instantaneous snapshots (top halves of individual images) and mean shots (bottom halves of individual images) of methane concentration of the four burning cases. From left to right, top to bottom: SwB1: premixed, non-swirling, SwB3: premixed, swirl-flow-ratio $=0.33$, SwB5: stratification ratio

$=1.0 / 0.5=2$, non-swirling and SwB7: stratification ratio $=1.0 / 0.5=2$, swirl-flow-ratio $=0.33$

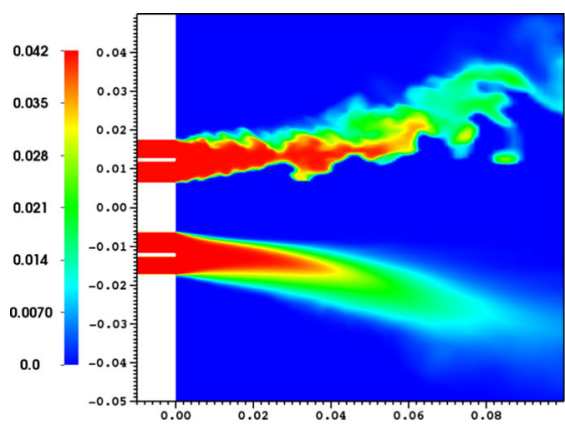

(a) SwB1

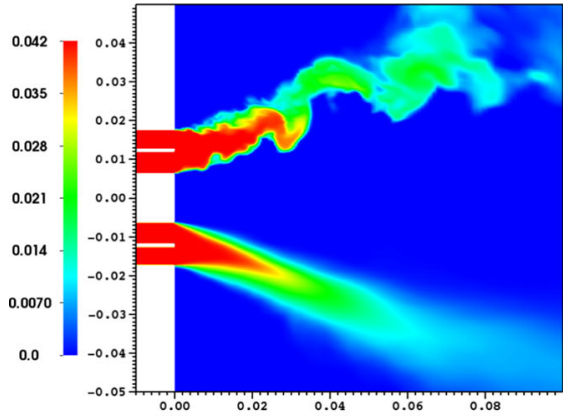

(b) SwB3

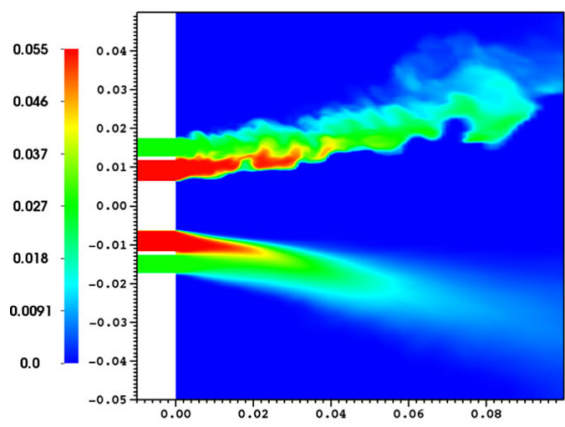

(c) SwB5

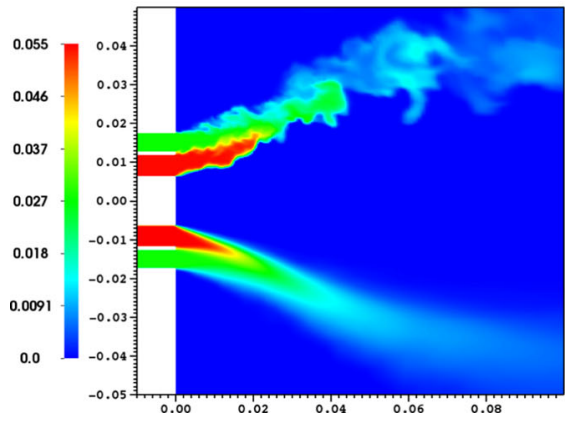

(d) $\mathrm{SwB} 7$ 
measurements at $2 \mathrm{~mm}$ above the bluff body are imposed on the generated turbulence and applied to the domain inlets. No turbulence was added to the co-flow. A detailed description of the method can be found in [28]. The species' concentrations at the inlets are assumed to be homogeneous. A detailed augmented reduced version of the GRI 3.0 mechanism, [29] with 15 reaction steps and 19 species is employed for the chemistry. For the burning cases eight stochastic fields are utilised to represent the $s g s$ contributions. The simulations are initialised with a single stochastic field and a spark model is used to initiate combustion, after which the simulations are allowed to settle before switching to 8 stochastic fields and eventually gathering statistics over the duration of at least 2 full domain flow through times based on the averaged inlet velocity of the inner annulus.
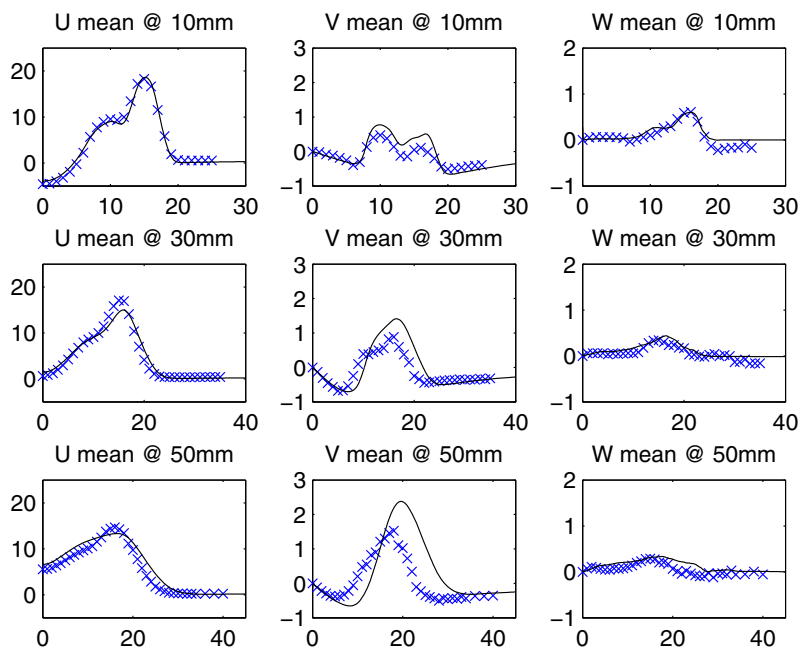

(a) Profiles of mean velocity components
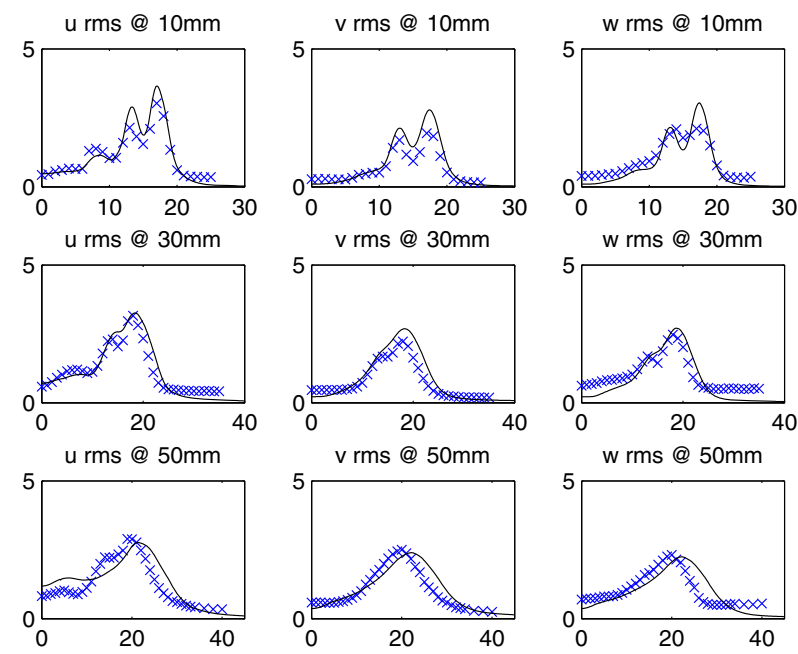

(b) Profiles of rms velocity components

Fig. 6 Comparison of axial (U), radial (V) and tangential (W) mean and rms (u,v,w) velocities for the stratified burning, non-swirling case, SwB1 


\section{Results and Discussion}

Results for the mean and fluctuating velocities of both the isothermal and burning cases as well as temperature and major species for the burning cases are presented at three measurement locations downstream of the bluff body: 10, 30 and $50 \mathrm{~mm}$. The isothermal results also include a comparison of data at $2 \mathrm{~mm}$ downstream of the bluff body.

Figures 3 and 4 compare simulation results (solid line) with experimental data (crosses) for all mean and fluctuating velocity components of the isothermal non-swirling, cSwB1, and highly swirling, cSwB3, cases. A small under prediction of the swirl is observed in
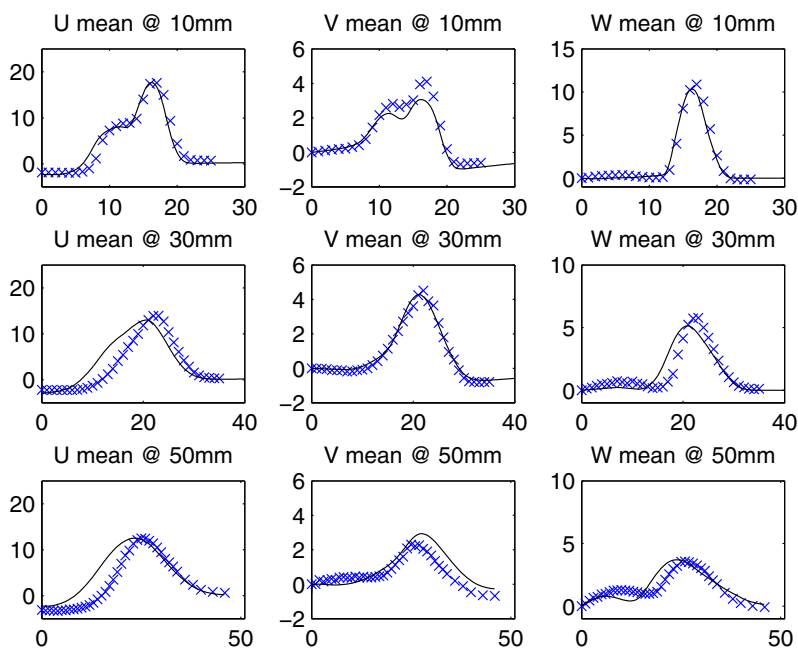

(a) Profiles of mean velocity components
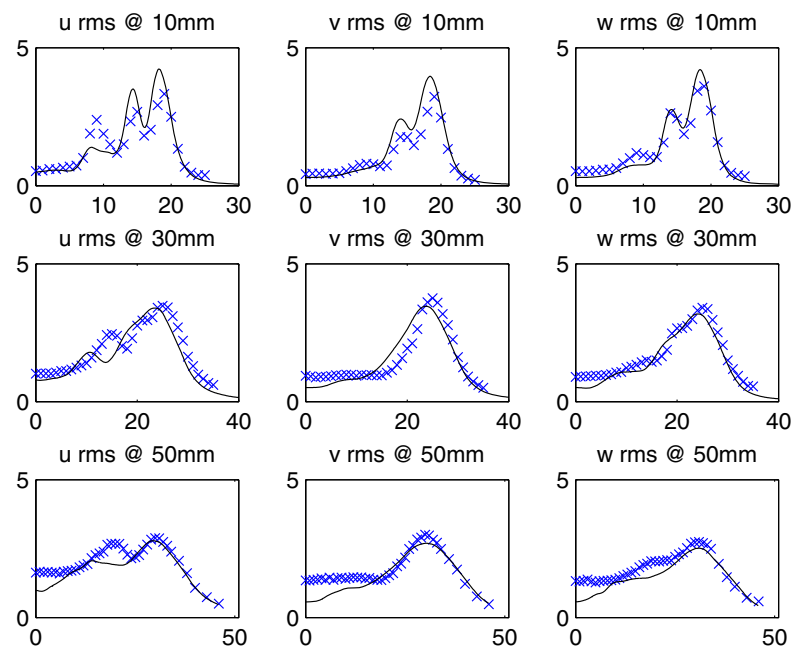

(b) Profiles of rms velocity components

Fig. 7 Comparison of axial (U), radial (V) and tangential (W) mean and rms (u,v,w) velocities for the stratified burning, swirling case, SwB3 
Fig. 4, likely due to loss of angular momentum by inclusion of part of the annuli. The results show good agreement for both cases and serve to support the choice of inflow conditions and mesh spacing.

Figures 6, 7, 8, 9 show mean and fluctuating velocities for the four burning cases SwB1, SwB3, SwB5 and SwB7 respectively. For both non-swirling cases, SwB1 and SwB5, the axial velocities are reproduced well, including the recirculation zone in the wake of the bluff body. The radial velocities (V) are slightly over-estimated at the furthest downstream location, which will be reflected in the scalar measurements. For the swirling cases, SwB3 and SwB7, the simulated tangential velocities (W) are slightly too low compared to measured
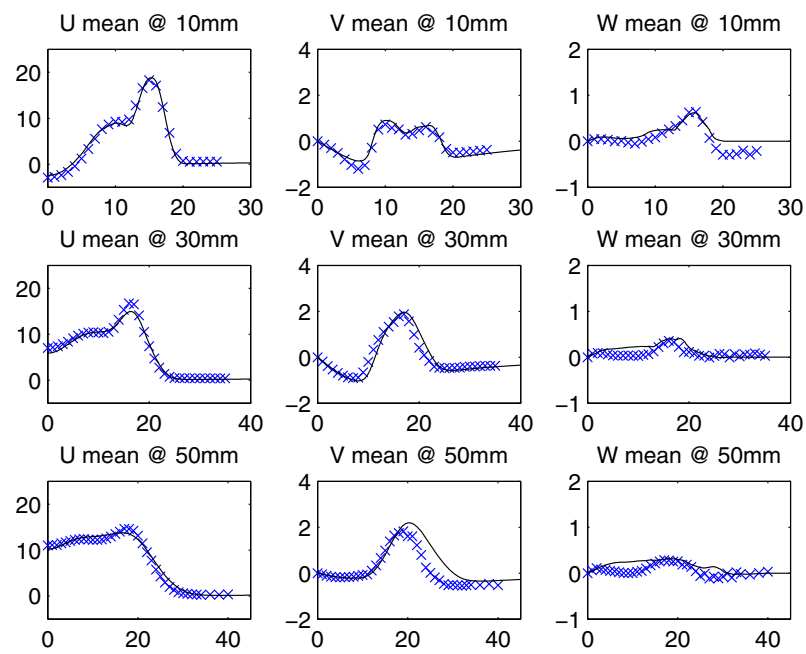

(a) Profiles of mean velocity components
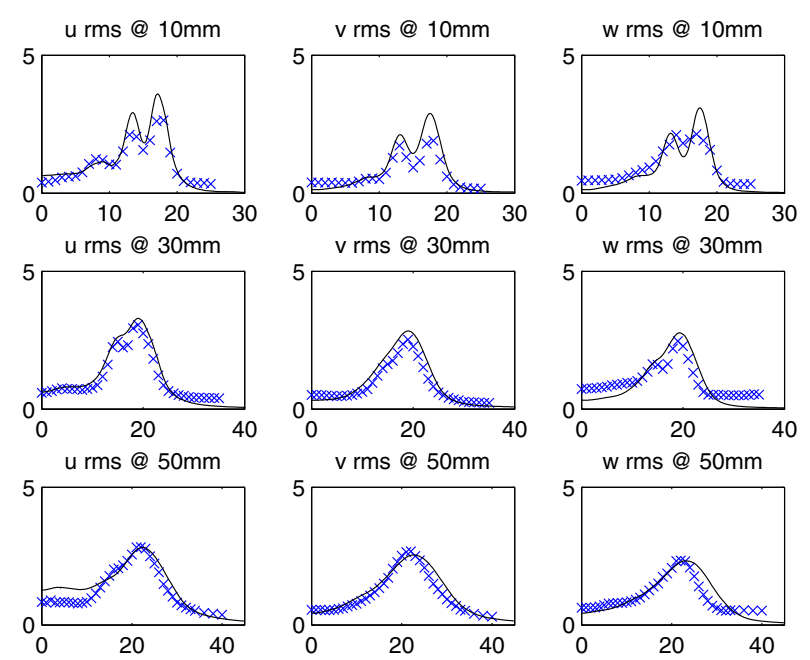

(b) Profiles of rms velocity components

Fig. 8 Comparison of axial (U), radial (V) and tangential (W) mean and rms (u,v,w) velocities for the stratified burning, non-swirling case, SwB5 

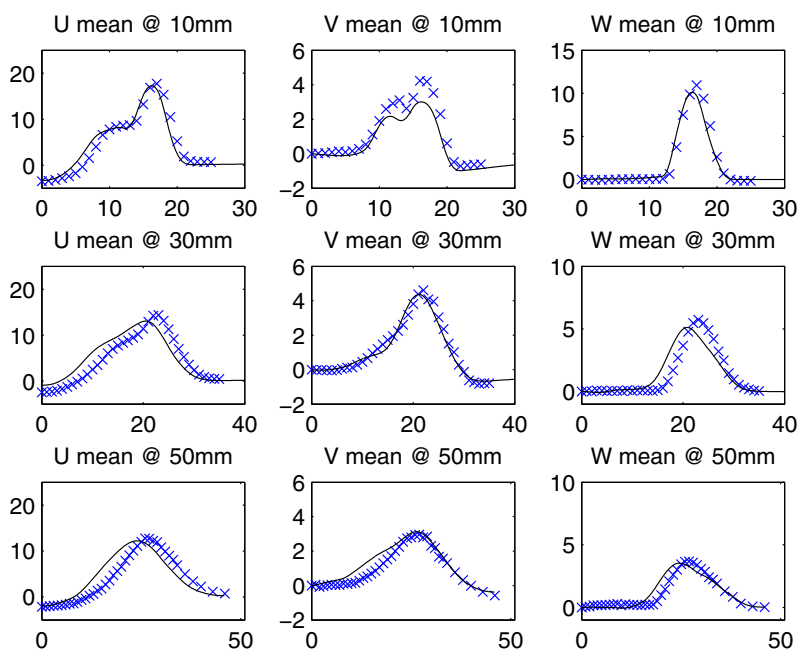

(a) Profiles of mean velocity components
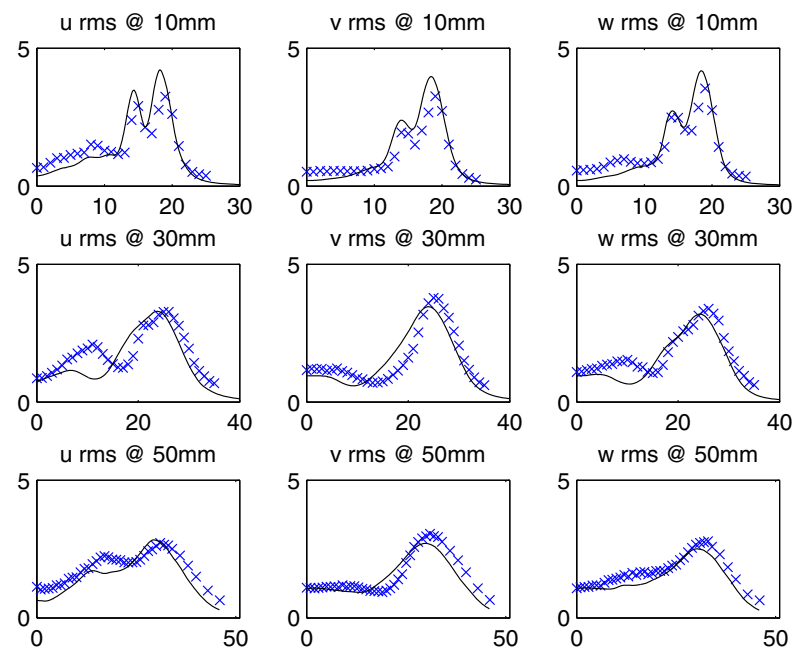

(b) Profiles of rms velocity components

Fig. 9 Comparison of axial $(\mathrm{U})$, radial $(\mathrm{V})$ and tangential $(\mathrm{W})$ mean and rms $(\mathrm{u}, \mathrm{v}, \mathrm{w})$ velocities for the stratified burning, swirling case, SwB7

values, likely due to the above mentioned choice of inlet conditions, while the simulated axial velocities in the burned mixture behind the flame front are somewhat too high. Overall, the trends and magnitudes are captured well and good agreement with the experimental data is achieved for both mean and fluctuating velocities. Any discrepancies will be discussed further in relation to the temperature and species results.

Figure 5 shows sample simulation results comprising instantaneous snapshots of methane concentration on the top half and mean shots on the bottom half of each individual image. The images are taken from a plane through the burner centre line. The top left image shows the premixed, non-swirling case, SwB1. The effect of swirl can be seen in the images to 
the right and the effect of stratification in the lower images. The fuel/air mixtures entering the domain are assumed to be homogeneous and downstream of the burner exit mixing of the outer annulus stream with the co-flow can be seen for all cases and mixing of the inner and outer annuli streams for the stratified cases. Combustion first takes place at the equivalence ratio of the inner (lower velocity, richer) stream, before the flame interacts with the shear layer and stratified mixture formed by the equivalence ratio and velocity gradients of the annuli streams. In this region, starting at $\sim 30 \mathrm{~mm}$, 'back- supported' burning from high towards low equivalence ratios takes place. High temperatures and increased presence of radicals and higher levels of turbulence from the outer annulus stream result in higher burning velocities supporting combustion into the lean mixture. Further downstream the flame interacts predominantly with the leaner mixture. Swirl increases the residence time,
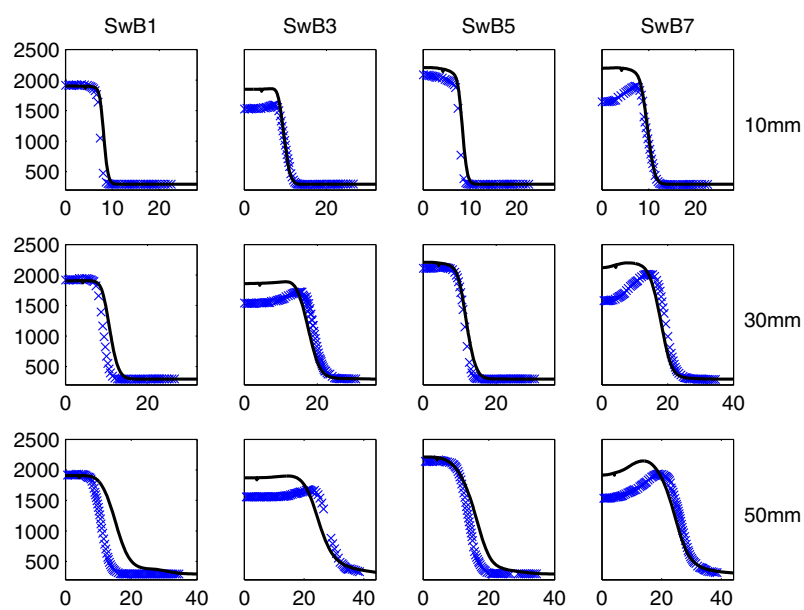

(a) Profiles of mean temperature
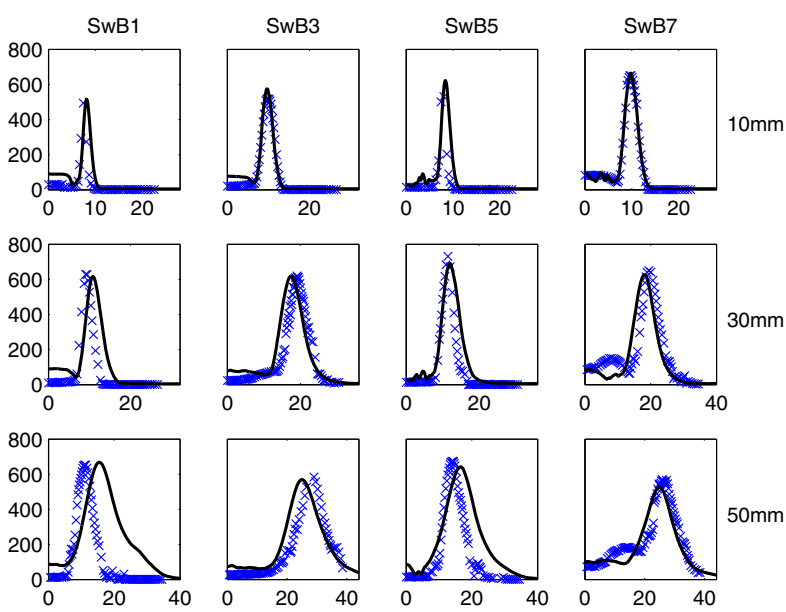

(b) Profiles of rms temperature

Fig. 10 Comparison of mean and rms temperature for all burning cases at 10, 30 and $50 \mathrm{~mm}$ 
shortens the flame and the same observations made for the non-swirling flames can be made closer to the bluff body.

In the region behind the bluff body the flow is well resolved with instantaneous ratios of $s g s$ to molecular viscosity $\mu_{s g s} / \mu$, not presented here, of less than 0.5 for the isothermal cases. For the burning cases there is no $s g s$ viscosity contribution due to re-laminarisation caused by the increase in molecular viscosity of the hot reaction products. The stochastic field method relies on the $s g s$ viscosity for both the stochastic and the micro-mixing terms and in well resolved or laminar regions with diminishing $s g s$ viscosity needs to recover the laminar flame propagation characteristics. The rearranged $p d f$ equation and the scaling of the sgs diffusive mixing term similar to that proposed by [12] provides a means way of achieving this at low viscosity ratios.
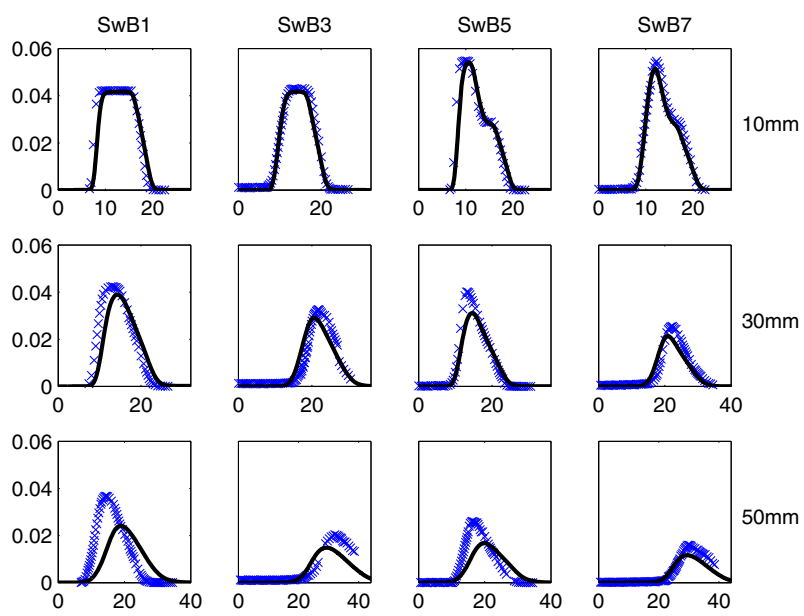

(a) Profiles of mean $\mathrm{CH}_{4}$ mass fractions
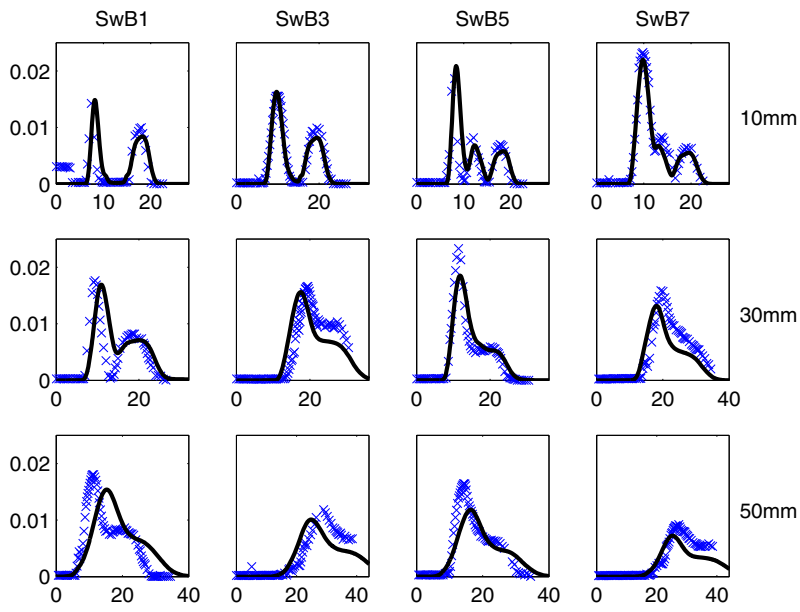

(b) Profiles of $\mathrm{rms} \mathrm{CH}_{4}$ mass fractions

Fig. 11 Comparison of mean and rms methane for all burning cases at 10, 30 and $50 \mathrm{~mm}$ 
Figure 10 compares the simulated profiles of mean and rms temperature with measurements for all burning cases. For the non-swirling cases, SwB1 and SwB5, agreement with the experimental results is good at the lower measurement location, whilst the simulated spreading rate is slightly too high at the downstream locations, mirroring the axial velocity results. For both swirling cases, SwB3 and SwB7, the spreading rate is well reproduced, however the temperature of the hot products close to the burner centre line is too high at all locations, which is in line with the higher axial velocities. Figures 11, 12, 13, 14 compare mean and rms profiles of $\mathrm{CH}_{4}, \mathrm{O}_{2}, \mathrm{CO}_{2}$ and $\mathrm{CO}$ for all cases. As expected the same observations made for temperature are evident for the species. Experimental profiles were measured across half of the burner at $30 \mathrm{~mm}$ and across the entire burner at $10 \mathrm{~mm}$ and $50 \mathrm{~mm}$ measurement locations. Agreement with the experimental data is improved when comparisons are made with both sides of the measurements profile taken across the entire burner,
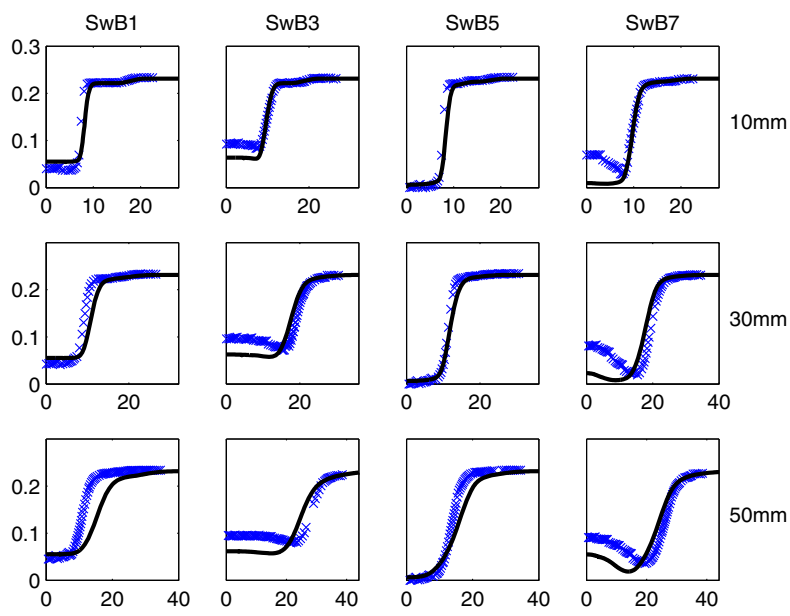

(a) Profiles of mean $\mathrm{O}_{2}$ mass fractions
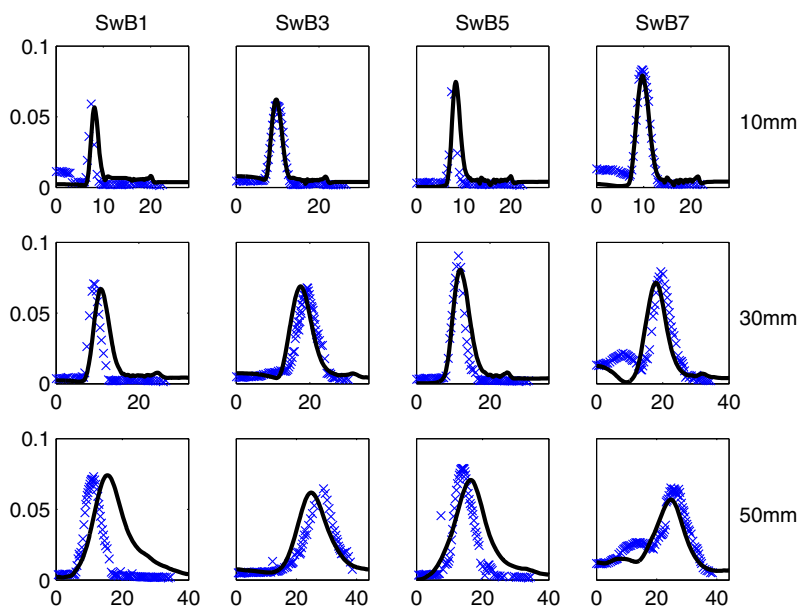

(b) Profiles of rms $\mathrm{O}_{2}$ mass fractions

Fig. 12 Comparison of mean and rms oxygen for all burning cases at 10, 30 and $50 \mathrm{~mm}$ 

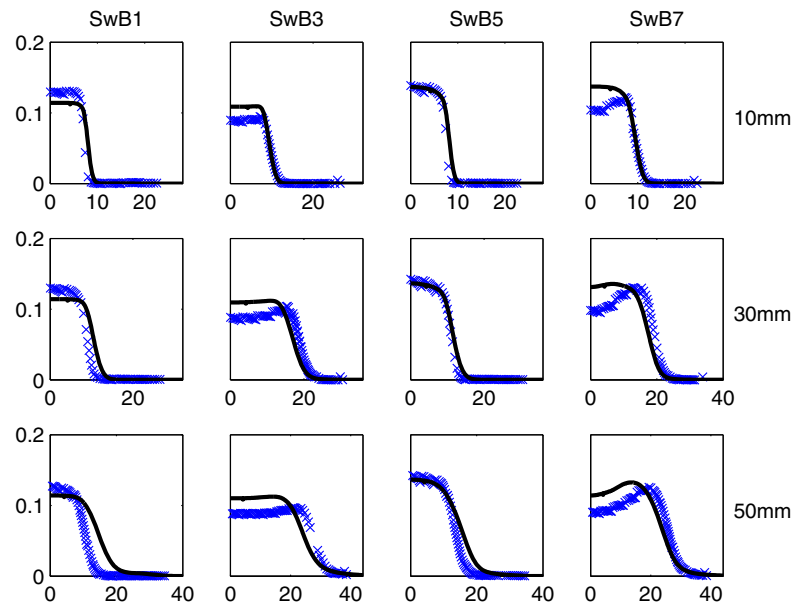

(a) Profiles of mean $\mathrm{CO}_{2}$ mass fractions
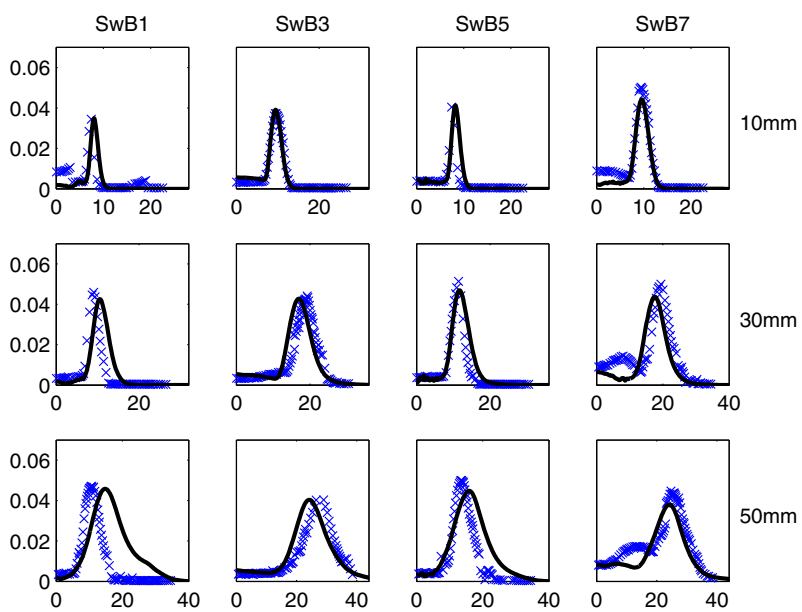

(b) Profiles of $\mathrm{rms}^{\mathrm{CO}}$ mass fractions

Fig. 13 Comparison of mean and rms carbon dioxide for all burning cases at 10, 30 and $50 \mathrm{~mm}$

thus taking some account of the asymmetry of the measured data. The simulations show the mixture to be fully burnt in the cases of the swirling flames, SWB3 and SWB7 whilst the mean $\mathrm{O}_{2}$ mass fractions are too low with the $\mathrm{CO}_{2}$ and temperatures being too high. This suggests that mixture is somewhat too rich (although fuel lean) the in the simulated flames compared with the measurements. Mixture fraction measurements would be needed to confirm this. For the flame SWB7 the simulated mean $C O$ mass fractions in the central region are also too high, probably for the same reason. For the non-swirling case SWB5 the simulated mean $C O$ levels are lower than those measured in the central region, although the reasons for this are unclear. Heat losses by conduction close to the bluff body and radiative heat transfer have been ignored in the present simulations, although the influences of these on the flames are expected to be very small. 

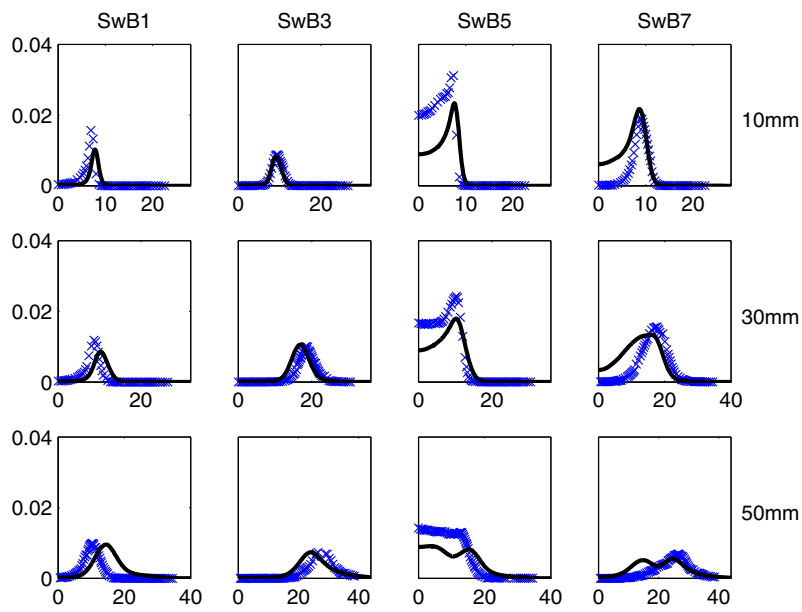

(a) Profiles of mean $\mathrm{CO}$ mass fractions
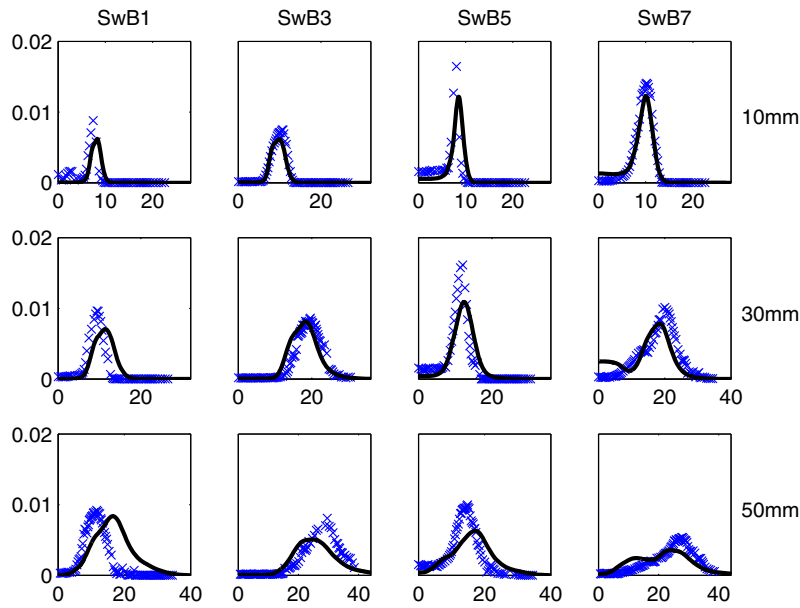

(b) Profiles of rms CO mass fractions

Fig. 14 Comparison of mean and rms carbon monoxide for all burning cases at 10, 30 and $50 \mathrm{~mm}$

\section{Conclusion}

The LES-pdf method with the stochastic field solution method with a reduced yet still detailed chemistry scheme has been applied to the premixed and moderately stratified, nonswirling and swirling flames of the Cambridge Stratified Swirl Burner. A modified version of the $p d f$ equation is used to ensure that the method is consistent with the limiting case of negligible sgs variations, an essential feature if measurements in low Reynolds number laboratory flames are to be reproduced. The simulated velocity fields and profiles are in excellent agreement with measured data for the isothermal and burning cases both with and without swirl. For the flames the overall agreement with measured profiles of $\mathrm{O}_{2}, \mathrm{CO}$, $\mathrm{CO}_{2}$ and $\mathrm{CH}_{4}$ mass fractions and temperature is good with maximum values being well reproduced by the simulations. There are some local discrepancies in the central regions of 
the two swirling flames, which, it is suggested, arise because the simulated mixture strength is too rich, although fuel lean, in this region. The flames studied all have relatively low Reynolds numbers and so the inclusion of more accurate transport properties (differential diffusion) may alleviate this and improve matters further.

Acknowledgments This investigation was generously supported by the UK Consortium on Turbulent Reacting Flows which granted access and computational time to and support for the UK national high performance computing facility ARCHER.

Open Access This article is distributed under the terms of the Creative Commons Attribution 4.0 International License (http://creativecommons.org/licenses/by/4.0/), which permits unrestricted use, distribution, and reproduction in any medium, provided you give appropriate credit to the original author(s) and the source, provide a link to the Creative Commons license, and indicate if changes were made.

\section{References}

1. Anselmo-Filho, P.: Experimental Investigations of Turbulent Stratified V-Flames Using Laser Diagnostics. $\mathrm{PhD}$ thesis, Cambridge University (2009)

2. Anselmo-Filho, P., Hochgreb, S., Barlow, R.S., Cant, R.S.: Experimental measurements of geometric properties of turbulent stratified flames. Proc. Combust. Inst. 32(2), 1763-1770 (2009)

3. Bruner, T., Jones, W.P., Marquis, A.J.: Large Eddy Simulation of turbulent premixed and stratified combustion using a sub-grid pdf method. In: 10th International Symposium on Engineering Turbulence Modelling and Measurements - ETMM10 (2014)

4. Sweeney, M.S.: Measurements of the structure of turbulent premixed and stratified methane/air flames (2011)

5. Sweeney, M.S., Hochgreb, S., Dunn, M.J., Barlow, R.S.: The structure of turbulent stratified and premixed methane/air flames I: Non-swirling flows. Comb. Flame 159(9), 2896-2911 (2012)

6. Sweeney, M.S., Hochgreb, S., Dunn, M.J., Barlow, R.S.: The structure of turbulent stratified and premixed methane/air flames II: Swirling flows. Comb. Flame 159(9), 2912-2929 (2012)

7. Nambully, S., Domingo, P., Moureau, V., Vervisch, L.: A filtered-laminar-flame pdf sub-grid scale closure for LES of premixed turbulent flames. part I: forMalism and application to a bluff-body burner with differential diffusion. Comb. Flame 161(7), 1756-1774 (2014)

8. Proch, F., Kempf, A.M.: Numerical analysis of the Cambridge stratified flame series using artificial thickened flame LES with tabulated premixed flame chemistry. Comb. Flame 161(10), 2627-2646 (2014)

9. Jones, W.P., Navarro-Martinez, S.: Large eddy simulation of auto-ignition with a sub-grid probability density function method. Comb. Flame 150(3), 170-187 (2007)

10. Mustata, R., Valiño, L., Jimenez, C., Jones, W.P., Bondi, S.: A probability density function Eulerian Monte Carlo field method for large eddy simulations: Application to a turbulent piloted methane/air diffusion flame (Sandia D). Comb. Flame 145(1-2), 88-104 (2006)

11. Jones, W.P., Prasad, V.N.: LES-Pdf simulation of a spark ignited turbulent methane jet. Proc. Combust. Inst. 33(1), 1355-1363 (2011)

12. Jones, W.P., Marquis, A.J., Prasad, V.N.: LES Of a turbulent premixed swirl burner using the Eulerian stochastic field method. Comb. Flame 159(10), 3079-3095 (2012)

13. Bulat, G., Jones, W.P., Marquis, A.J.: NO And CO formation in an industrial gas-turbine combustion chamber using LES with the Eulerian sub-grid PDF method. Combust. Flame 161(7), 1804-1825 (2014)

14. Germano, M., Piomelli, U., Moin, P., Cabot, W.: A dynamic sub-grid scale eddy viscosity model. (1991). doi:10.1063/1.857955

15. Piomelli, U., Liu, J..: Large Eddy Simulation of rotating channel flows using a localised dynamic model. Phys. Fluids 7(4), 893-848 (1995)

16. Gao, F., O'Brien, E.: A large eddy simulation scheme for turbulent reacting flows. Phys. Fluids A 5, 1282-1284 (1993)

17. Colucci, P., Jaberi, F., Givi, P., Pope, S.: Filtered density function for large eddy simulation of turbulent reacting flows. Phys. Fluids 10, 499-515 (1998)

18. Jaberi, F.A., Colucci, P.J., James, S., Givi, P., Pope, S.B.: Filtered mass density function for large-eddy simulation of turbulent reacting flows. J. Fluid Mech. 401, 85-121 (1999) 
19. Jones, W.P., Prasad, V.N.: Large Eddy Simulation of the Sandia Flame Series (D, E \&F). Eulerian Stoch. Field Method. Comb. Flame 157(9), 1621-1636 (2010)

20. Dopazo, C.: Probability density function approach for an axisymmetric heated jet: centerline evolution. Phys. Fluids 18, 397 (1975)

21. Dopazo, C.: Relaxation of initial probability density function in turbulent convection of scalar fields. Phys. Fluids 22(1), 20-30 (1979)

22. Dopazo, C., O'Brien, E.: Functional formulation of non-isothermal turbulent reactive flows. Phys. Fluids 17(1968) (1974)

23. Protter, M.H., Murray, H.: Maximum Principles in Differential Equations. Prentice-Hall, New Jersey (1967)

24. Gardiner C: Handbook of Stochastic Methods. Springer Verlag (1985)

25. Valiño, L., Mustata, R., Letaief, K.B.: Consistent Behaviour of Eulerian Monte Carlo fields at Low Reynolds Numbers Flow Turbines Combinatorial. (2015). doi:10.1007/s10494-015-9687-0

26. Barlow, R.S., Dunn, M.J., Sweeney, M.S., Hochgreb, S.: Effects of preferential transport in turbulent bluff-body-stabilised lean premixed CH4/air flames. Combust. Flame 159(8), 2563-2575 (2012)

27. Zhou, R.: Turbulence parameters for non-reacting conditions of Cambridge Stratified Swirl Burner. Technical Report, Cambridge University/A-TURBO/TR.135, http://www.dspace.cam.ac.uk/handle/ 1810/24325 (2014)

28. Klein, M., Sadiki, A., Janicka, J.: A digital filter based generation of inflow data for spatially developing direct numerical or large eddy simulations. J. Comput. Phys. 186(2), 652-665 (2003)

29. Sung, C.J., Law, C.K., Chen, J.Y.: Augmented reduced mechanisms for NO emission in Methane oxidation. Comb. Flame 125, 906-919 (2001) 